\title{
Made-to-Measure N-body Modeling of the Milky Way Galaxy
}

\section{Ortwin Gerhard}

\author{
Max-Planck-Institut für Ex. Physik, Giessenbachstrasse 1, D-85748 Garching, Germany \\ email: gerhard@mpe.mpg.de
}

\begin{abstract}
In this talk a brief introduction is given to made-to-measure particle methods and their potential use for modeling the Milky Way Galaxy.
\end{abstract}

\section{Made-to-Measure N-body models}

The term "Made-to-Measure" (M2M) for a dynamical model reproducing a set of observational data for a galaxy was coined by Syer \& Tremaine (1996) (hereafter ST). M2M models can be based on distribution functions, moments, orbits, or particles, but in particular ST described an algorithm for constructing N-body equilibrium models, based on the idea of adjusting the masses or weights of the particles until the system agreed with a prescribed density distribution. The central part of their algorithm is the "forceof-change" (FOC) equation through which the particle weights are adjusted according to the mismatch of model and target density observables. Other important ingredients include a time smoothing to reduce particle noise and an entropy term to reduce large fluctuations in the weights.

As a first practical application of this method Bissantz, Debattista \& Gerhard (2004) constructed a dynamical model for the Milky Way's barred bulge and disk, constraining the projected density map. However, the ST algorithm is not well-suited for mixed density and kinematic observables, and it does not allow a proper treatment of observational errors. A modified $\chi^{2} \mathrm{M} 2 \mathrm{M}$ algorithm which resolved both these problems was introduced by De Lorenzi et al. (2007) (DL+07) who also demonstrated the potential of the method by constructing particle models for spherical, axisymmetric, triaxial and rotating target systems. Their implementation NMAGIC has since been used by De Lorenzi et al. $(2008,2009)(\mathrm{DL}+08, \mathrm{DL}+09)$ to construct dynamical models of elliptical galaxies based on photometry, Sauron, slit and planetary-nebula velocity data, and thus to explore the distribution of dark matter in these galaxies. For modeling the discrete velocities, De Lorenzi et al. (2008) introduced a likelihood scheme into the FOC equation, and they also added a separate equation for adjusting the mass-to-light ratio of the stellar system simultaneously with the observables.

A modified M2M method was introduced by Dehnen (2009). Rather than timeaveraging the moments of the N-body system as ST and DL +07 , he considered timeaveraging the merit function, resulting in a second-order equation for adjusting the particle weights. This contains a linear damping term that acts to maximize the merit function. Writing the equations in dimensionless time (in units of orbital period) also made it possible to achieve a uniform adjustment rate per orbital time for the particle weights, despite the large range of orbital time-scales inherent in an N-body system. Dehnen (2009) used this algorithm to construct triaxial equilibrium target systems. 


\section{Comparisons with Schwarzschild and direct N-body methods}

M2M particle models include aspects of both Schwarzschild orbit superposition methods and direct N-body simulations. When the gravitational potential of the target system is held fixed, the process of finding a distribution of particle weights to match a prescribed density field or set of kinematic observables for a galaxy, is closely related to the process of finding a distribution of orbital weights in Schwarzschild's method to fit the same constraints. Conversely, when the adjustment of particle weights is switched off and the potential is allowed to evolve, M2M particle codes reduce to N-body methods. E.g., in the work of DL+07 several equilibria are found in the first limit, while the stability of the final galaxy models in DL+08 and DL+09 is tested in the second limit. Of course, the strength of the M2M particle methods is to self-consistently evolve the particle weights and their potential simultaneously while approaching the target data (see applications in DL+08). This is perhaps the greatest advantage over Schwarzschild and N-body techniques. However, this is most useful when the particles trace the mass. Because there is no direct connection between observables and the global gravitational potential, external dark-matter potentials have still to be explored one by one. Compared to Schwarzschild and N-body techniques, M2M modeling is still in an early stage, and improvements of both the techniques and the efficiency of the method are needed.

\section{M2M modeling for the Milky Way}

The goal of dynamical models for the Milky Way is to uncover the fossil record of its assembly history, as described by, for example, the orbital distribution of stars of different metallicities or other population parameters. Because of the enormous detail expected from future observations, particularly from Gaia, it is likely that interesting (sub)structure in the models would be visible in the data. Thus techniques with a minimum of simplfying assumptions such as M2M may be well-suited to understand what these data will be telling us.

M2M work to date has been confined to the inner Galaxy; see Bissantz, Debattista \& Gerhard (2004) and Debattista et al. (in preparation). These models have successfully reproduced as diverse observables as densities, radial velocity histograms and microlensing event time-scale distribution, but a comprehensive bulge model is still pending.

Modeling the nearby Galactic disk has not been tried yet. This has the problem that only a small fraction of the particles from any such model will be seen in magnitude limited surveys; see e.g., Brown et al. (2005). However, this may actually not be such a large problem for M2M methods such as NMAGIC. The time averaging of the observables allows suppressing the particle noise in the model observables by a factor 10-100, which eases the comparison with solar neighbourhood data.

In conclusion, made-to-measure modeling for the Milky Way has little history, but a lot of promise, and so there is a lot of work to do!

\section{References}

Bissantz, N., Debattista, V. P., \& Gerhard, O. 2004, ApJ, 601, L155

Brown, A. G. A., Velázquez, H. M., \& Aguilar, L. A. 2005, MNRAS, 359, 1287

Dehnen, W. 2009, MNRAS, 395, 1079

De Lorenzi, F., Debattista, V. P., Gerhard, O., \& Sambhus, N. 2007, MNRAS, 376, 71

De Lorenzi, F., Gerhard, O., Saglia, R. P., \& Sambhus, N., et al. 2008, MNRAS, 385, 1729

De Lorenzi, F., Gerhard, O., Coccato, L., \& Arnaboldi, M., et al. 2009, MNRAS, 395, 76

Syer, D., Tremaine, S. 1996, MNRAS, 282, 223 